\title{
Asiatic acid protests against myocardial ischemia/reperfusion injury via modulation of glycometabolism in rat cardiomyocyte
}

This article was published in the following Dove Press journal:

Drug Design, Development and Therapy

\section{Yang Dai ${ }^{1, *}$ \\ Ziwei Wangl,* \\ Minxue Quan' \\ Yanni Lv' \\ Yunman $\mathrm{Li}^{3}$ \\ Hong-Bo Xin' \\ Yisong Qian'}

'Institute of Translational Medicine, Nanchang University, Nanchang 33003I, China; ${ }^{2}$ Department of Pharmacy, The First Affiliated Hospital of Nanchang University, Nanchang 330046, China; ${ }^{3}$ Department of Physiology, China Pharmaceutical University, Nanjing 210009, China

*These authors contributed equally to this work
Correspondence: Yisong Qian; Hong-Bo Xin Institute of Translational Medicine, Nanchang University, 1299 Xuefu Avenue, Nanchang 33003I, China Email qianyisong@ncu.edu.cn; hongboxin@yahoo.com
Background: Asiatic acid is a reported glycogen phosphorylase inhibitor derived from the tropical medicinal plant Centella asiatica and exhibits myocardial protection both in vivo and in vitro. The purpose of this study was to evaluate the effects of asiatic acid on myocardial ischemia/reperfusion (MI/R) injury and investigate the underlying mechanisms associated with the modulation of glycometabolism in cardiomyocyte.

Materials and methods: The rats were subjected to MI/R with or without asiatic acid pretreatment. The cardiac function indexes, the size of myocardial infarction, and plasma lactate dehydrogenase $(\mathrm{LDH})$ and creatine kinase $(\mathrm{CK})$ activities were detected. Cardiomyocyte apoptosis was analyzed by TUNEL assay. The Akt/GSK-3 $\beta$ activation was measured by Western blot. The glycogen content, plasma glucose and lactate concentrations were determined following MI/R. The mRNA and protein levels of PPAR $\gamma$ and GLUT4 were determined by real-time PCR and Western blot, respectively.

Results: Asiatic acid pretreatment significantly improved the cardiac function indexes, attenuated the size of myocardial infarction, reduced LDH and CK activities, and suppressed cardiomyocyte apoptosis after MI/R. Asiatic acid activated Akt/GSK-3 $\beta$ signal pathway in the myocardium following MI/R injury. In addition, asiatic acid effectively suppressed MI/R-induced glycogen breakdown and inhibited the elevation of plasma glucose and lactate concentrations. Asiatic acid treatment increased PPAR $\gamma$ expression at both mRNA and protein levels, and promoted the translocation of GLUT4 to plasma membrane after MI/R insult. However, the effects mediated by asiatic acid on glycometabolism and GLUT4 translocation were reversed by the administration of LY294002, the Akt inhibitor.

Conclusion: These findings demonstrated that asiatic acid exerts beneficial effects on MI/R injury in rats. This protection may be related to the modulation of glycometabolism via Aktdependent GLUT4 translocation and PPAR $\gamma$ activation in ischemic cardiomyocyte.

Keywords: asiatic acid, myocardial ischemia/reperfusion, glycometabolism, Akt, PPAR $\gamma$

\section{Introduction}

Acute myocardial ischemia/reperfusion (MI/R) injury is a major cause of death and provokes irreversible metabolic changes and exacerbated tissue injury. ${ }^{1}$ Under ischemic conditions, myocardial oxidative metabolism is suppressed while glycolysis is stimulated as the important source of ATP generation to ensure cell survival. ${ }^{2}$ However, the excessive glycolysis leads to ATP depletion along with the accumulation of lactate and $\mathrm{H}^{+}{ }^{3}$ The process may continue during reperfusion and impairs the $\mathrm{Ca}^{2+}$ uptake capacity of the sarcoplasmic reticulum and the abilities of oxygen free radical scavenging system, which further disturbs cellular energetics and triggers the irreversible 
apoptotic and necrotic cell death. ${ }^{4}$ Therefore, regulation of cardiac energy substrate metabolism by reducing myocardial anaerobic glycolysis has emerged as an effective therapeutic intervention to protect against $\mathrm{MI} / \mathrm{R}$ injury.

Glycogen phosphorylase (GP) is a key component for controlling the plasma glucose levels via depolymerization of glycogen to glucose-1-phosphate. ${ }^{5}$ Pharmacological GP inhibitors have been developed and studied as a promising target for the treatment of hyperglycemia associated with type 2 diabetes. ${ }^{6,7}$ There is also evidence that GP inhibitors may be cardioprotective by inhibition of glycogenolysis and the associated proton production, thereby reducing myocardial ischemic injury. ${ }^{8}$ Asiatic acid, a natural pentacyclic triterpene derived from Centella asiatica, has been reported as a potent GP inhibitor that binds to the allosteric activator site where the physiological activator AMP binds ${ }^{9}$ and exerts hypoglycemic effects in a spontaneous type 2 diabetic animal model. ${ }^{10}$ Our previous work has demonstrated that asiatic acid exhibits cardioprotective effects against oxygen-glucose deprivation/ reoxygenation-induced apoptosis in vitro. ${ }^{11}$ However, whether asiatic acid prevents myocardial ischemia injury in in vivo models and the underling mechanisms mediated by asiatic acid remain largely unknown.

In this study, we investigated the protective effects of asiatic acid in a MI/R model of rats. The key targets and signal pathways associated with glycometabolism during ischemia were detected, with the purpose of elucidating the mechanism by which asiatic acid protects against MI/R injury.

\section{Materials and methods}

\section{Reagents}

Asiatic acid and LY294002 were purchased from SigmaAldrich Co. (St Louis, MO, USA). The anti-Akt and anti-phosphor-Akt antibodies were obtained from Santa Cruz Biotechnology, Inc. (Santa Cruz, CA, USA); the antiGSK-3 $\beta$ and anti-phospho-GSK-3 $\beta$ (Ser9) antibodies were from Cell Signaling Technology, Inc. (Danvers, MA, USA); the anti-GLUT4 and anti-PPAR $\gamma$ antibody was from Boster Biological Technology, Ltd. (Wuhan, China); and the antiglyceraldehyde phosphate dehydrogenase (GAPDH) antibody was obtained from KangChen Bio-tech Inc. (Shanghai, China).

\section{Animal treatment and $M I / R$ induction}

Male SD rats from 8 to 10 weeks were purchased from Zhejiang Experimental Animal Center (Zhejiang certificate no 2014-0001) and were housed in the central laboratory of The First Affiliated Hospital of Nanchang University with a 12-hour light-dark cycle, optimal temperature and humidity, filtered water, and appropriate nutrient feed. All the experimental procedures were carried out in accordance with the National Institutes of Health Guidelines. All experimental protocols were approved by the Institutional Animal Care and Use Committee of Nanchang University (approval number: 2016-001). All efforts were made to minimize animal suffering and reduce the number of animals used.

Age-matched rats were randomly assigned to control or experimental groups with $n=8$ for each group. Animals were administered intragastrically with vehicle or various doses of asiatic acid $(2.5,5$, and $10 \mathrm{mg} / \mathrm{kg}$, suspended in $0.5 \%$ sodium carboxymethyl cellulose) 0.5 hours before MI/R. The dosage chosen was based on previous studies ${ }^{12}$ as well as our preliminary experiments to ensure both the efficacy and safety. In another set of experiments, asiatic acid-treated rats received LY294002 (30 mg/kg intraperitoneally), a PI3K/Akt inhibitor, 0.5 hours before asiatic acid administration. ${ }^{13} \mathrm{MI} / \mathrm{R}$ was induced as previously described, with some modifications. ${ }^{14}$ Rats were anesthetized with chloral hydrate $(400 \mathrm{mg} / \mathrm{kg}$ intraperitoneally), and then a $20 \mathrm{G}$ intravenous catheter was inserted and a rodent ventilator was used for ventilation. A left thoracotomy was performed. The left anterior descending (LAD) coronary artery was visualized and ligated using 7-0 silk suture around fine polyethylene-10 tubing with a slip knot. Rats were subjected to 1 hour of LAD ischemia followed by 24 hours of reperfusion. Then the Vevo 2,100 System (VisualSonics Inc., Toronto, ON, Canada) was used to record the left ventricular end diastolic volume (LVEDV) and the left ventricular end systolic volume (LVESV). The heart rate $(\mathrm{HR})$ and left ventricular pressure maximal rate of rise $\left(\mathrm{LV} \mathrm{dp} / \mathrm{dt}_{\max }\right)$ and decline ( $\mathrm{LV} \mathrm{dp} / \mathrm{dt}_{\text {min }}$ ) were recorded by using the PowerLab physiological system (ADInstruments, Castle Hill, NSW, Australia). All measurements were covered for at least five consecutive cardiac cycles. After re-occluding the LAD and intravenous injection of Evans blue dye $(2 \% \mathrm{w} / \mathrm{v})$ to stain the area at risk (AAR), the heart was removed and cut into four horizontal slices. Sections were then incubated in a $p$-nitro-blue tetrazolium $(0.2 \% \mathrm{w} / \mathrm{v})$ solution for 20 minutes and digitally photographed. The left ventricular area, AAR, and infarct area for each slice were determined by computerized planimetry, respectively, using Image Pro Plus 6.0 (Media Cybernetics, Bethesda, MD, USA), and the infarct size was expressed as a percentage of the AAR.

\section{Determination of plasma lactate dehydrogenase (LDH) and creatine kinase (CK) activities}

At the end of the reperfusion, blood samples were collected and centrifuged for plasma separation $(1,000 \times g$, 
10 minutes, $\left.4^{\circ} \mathrm{C}\right)$. Plasma $\mathrm{LDH}$ and $\mathrm{CK}$ activities were measured using commercial assay kits (Nanjing Jiancheng Bioengineering Institute, Nanjing, China) according to the manufacturer's instructions.

\section{TUNEL assay}

The rats were anesthetized and perfused with $0.9 \%$ saline, followed by $4 \%$ paraformaldehyde, at 24 hours after reperfusion. The histological sections were prepared and stained according to the manufacturer's instructions (KeyGen Biotech Co Ltd, Nanjing, China). The TUNEL-positive cells in the peri-infarct zone of the heart were captured using a quantitative digital analysis system (NIH Image 1.6; National Institutes of Health, Bethesda, MA, USA) and counted using Image Pro Plus 6.0. A total of five fields in the peri-infarct areas on each slide were randomly examined.

\section{Isolation of heart plasma membrane fraction}

Heart plasma membrane (PM) fraction was prepared as described previously. ${ }^{15}$ Ventricle tissue was homogenized in buffer $\mathrm{A}\left(10 \mathrm{mM} \mathrm{NaHCO}_{3}\right.$ and $\left.5 \mathrm{mM} \mathrm{NaN}_{3}\right)$ and then centrifuged at $7,000 \times g$ for 20 minutes. The pellet was resuspended in buffer $\mathrm{B}(10 \mathrm{mM}$ Tris- $\mathrm{HCl}, \mathrm{pH} 7.4)$ and centrifuged at $200 \times g$ for 20 minutes. The supernatant was gently layered on top of a $20 \%(\mathrm{v} / \mathrm{v})$ Percoll gradient in buffer C (255 mM sucrose, $10 \mathrm{mM}$ Tris-HCl, $\mathrm{pH} 7.4$, and $2 \mathrm{mM}$ EDTA) and centrifuged at 55,000× $g$ for 1 hour. The band at a density of 1.030 was aspirated and pelleted by centrifugation at $170,000 \times g$ for 1 hour and resuspended in buffer $\mathrm{C}$ as PM solution. Protein concentration of PM solution was determined with bicinchoninic acid protein assay. GLUT4 levels in PM were determined by Western blot.

\section{Western blot}

The ventricle tissue was collected and lysed in RIPA lysis buffer. Equal amounts of protein per sample were loaded in each lane, separated by SDS-PAGE, and transferred to polyvinylidene fluoride membranes. The membranes were blocked with skimmed milk for 1 hour, washed in Tris buffered saline containing $0.1 \%$ Tween-20 (TBST), and incubated overnight with the primary antibodies. After washing three times with TBST, the membranes were incubated for 1 hour at room temperature with horseradish peroxidase-conjugated goat anti-rabbit or anti-mouse IgG. Bands were visualized using the SuperSignalWest Pico Chemiluminescent Substrate Trial Kit (Pierce, Rockford, IL, USA). Images were taken using the ChemiDoc XRS system with Quantity One software (Bio-Rad, Richmond, CA, USA).

\section{Real-time PCR}

Total RNA was isolated from ventricle tissue using the TRIzol $^{\text {TM }}$ reagent (Life Technologies, Carlsbad, CA, USA) according to the manufacturer's instructions. One microgram of total RNA was reverse-transcribed using a One Step PrimeScript ${ }^{\mathrm{TM}}$ RT-PCR Kit (Takara, Dalian, China) with a thermocycler. Real-time PCR was performed using the ABI 7500 sequence detection system with a reaction mixture that consisted of SYBR Green $2 \times$ PCR Master Mix (Thermo Fisher Scientific, Waltham, MA, USA), cDNA template, forward primer, and reverse primer. Primer sequences were as follows: 5'-CCAACTTCGGAATCAGCTCTGT-3' and 5'-GGAGAAATCAACCGTGGTAAAGG-3' (PPAR $\gamma$ ), 5'-CATTCTCGGACGGTTCCTCAT-3' and 5'-GCGATTTCTCCCACATACATAGG-3' (GLUT4), and 5'-TGGCCTCCAAGGAGTAAGAAAC-3' and 5'-GGCCTCTCTCTTGCTCTCAGTATC-3' (GAPDH). The PCR protocol consisted of 40 cycles of denaturation at $95^{\circ} \mathrm{C}$ for 15 seconds followed by $60^{\circ} \mathrm{C}$ for 1 minute to allow extension and amplification of the target sequence. Data were analyzed using ABI 7500 sequence detection system software. The amount of mRNA was normalized to GAPDH using the $2^{-\triangle \Delta C T}$ method. The results were from three independent experiments performed in triplicate.

\section{Determination of plasma glucose and lactate concentrations}

Blood samples were collected after 1 hour of ischemia and 24 hours of reperfusion, respectively, followed by centrifugation and collection of the plasma. Plasma glucose and lactate concentrations were determined using commercial kits (Nanjing Jiancheng Bioengineering Institute). The absorbance value was detected at $492 \mathrm{~nm}$ for glucose and at $530 \mathrm{~nm}$ for lactate.

\section{Measurement of myocardial glycogen content}

Myocardial glycogen content was measured as described previously. ${ }^{8}$ At the end of the 1-hour period of ischemia and 24 hours of reperfusion, hearts were removed from the animals and rapidly perfused with ice-cold saline. A total of $1.5 \mathrm{~mL}$ of $30 \% \mathrm{KOH}$ was saturated with $\mathrm{Na}_{2} \mathrm{SO} 4$ and immersed in a boiling water bath for 30 minutes before glycogen was assayed using a commercial kit (Nanjing Jiancheng Bioengineering Institute). Samples were then immediately cooled at $4^{\circ} \mathrm{C}$ and were detected at $620 \mathrm{~nm}$ in the ultraviolet-visible spectrophotometer. 


\section{Statistical analysis}

All values are expressed as the mean \pm SD of at least three independent preparations. Differences among the groups were compared using one-way ANOVA analysis followed by a Tukey post hoc test. A difference with $P<0.05$ was considered statistically significant.

\section{Results}

\section{Asiatic acid improves cardiac function} and reduces myocardial infarction size following $M \mathrm{II} / \mathrm{R}$

The levels of LVEDV, LVESV, HR, LV dp/dt ${ }_{\text {max }}$, and LV $\mathrm{dp} / \mathrm{dt}_{\min }$ were measured following 1 hour of myocardial ischemia and 24 hours of reperfusion for evaluating the cardiac function in rats. Compared with the sham group, the LVEDV and LVESV obviously increased after MI/R. However, asiatic acid at $2.5-10 \mathrm{mg} / \mathrm{kg}$ treatment reduced LVEDV and LVESV in a dose-dependent manner (Figure 1A

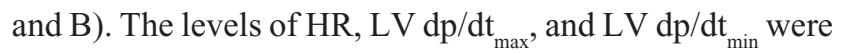
significantly decreased in the MI/R group compared with the sham group, whereas asiatic acid pretreatment significantly increased these parameters following MI/R (Figure 1C-E), suggesting that asiatic acid effectively improved heart function in ischemic rats.

After 24 hours of reperfusion, rats were killed and the hearts were isolated for infarction size assay. Compared with the sham group, the myocardial infarction size was significantly increased in the vehicle group. Asiatic acid elicited a dose-dependent reduction in infarct size after $M I / R$, and the maximum reduction in infarct size was achieved with $10 \mathrm{mg} / \mathrm{kg}$ asiatic acid treatment (Figure 1F). The abovementioned data indicated that asiatic acid is protective against $\mathrm{MI} / \mathrm{R}$ injury.

\section{Asiatic acid reduced $\mathrm{LDH}$ and $\mathrm{CK}$ activities in $M I / R$ rats}

The activity of LDH in MI/R rats was significantly higher than that in the Sham rats, and administration of asiatic acid reduced LDH activity in a dose-dependent manner (Figure 2A). MI/R induced a threefold increase in serum
A

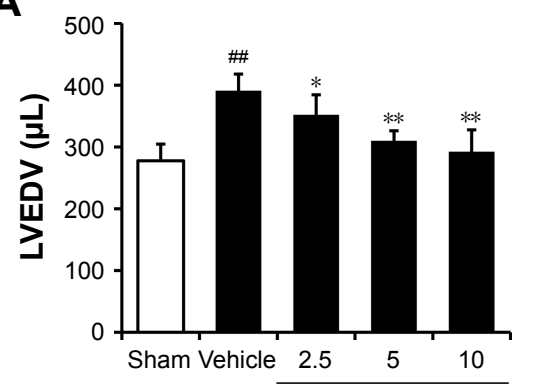

Asiatic acid (mg/kg)

D

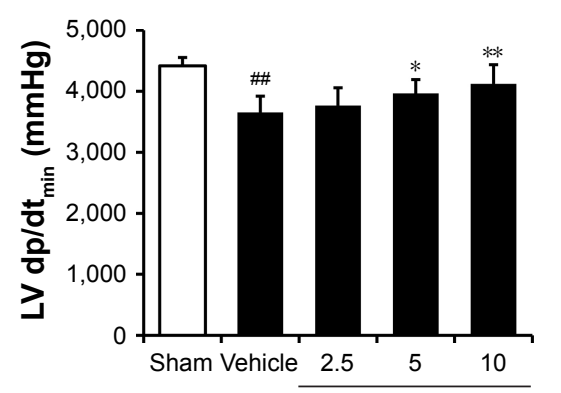

Asiatic acid (mg/kg)
B

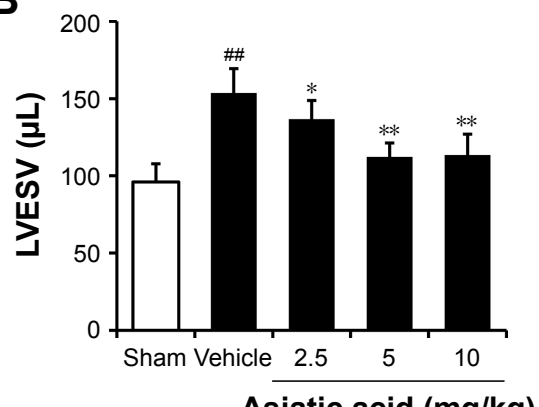

Asiatic acid (mg/kg)

E

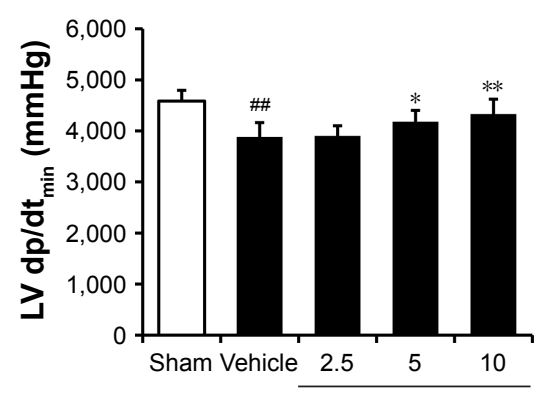

Asiatic acid $(\mathrm{mg} / \mathrm{kg})$

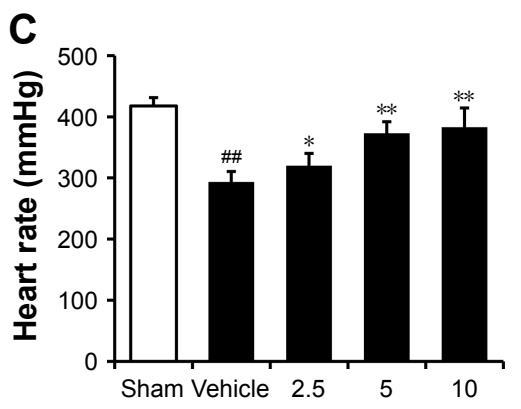

Asiatic acid (mg/kg)

$\mathbf{F}$

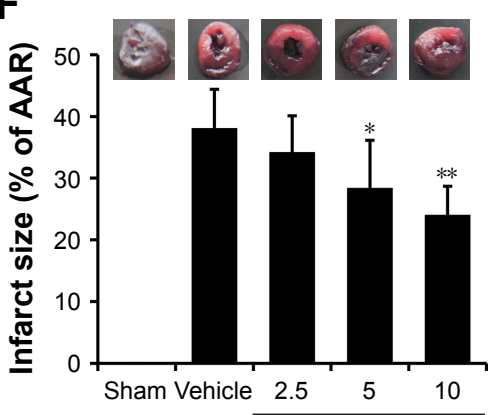

Asiatic acid $(\mathrm{mg} / \mathrm{kg})$

Figure I Asiatic acid improves cardiac function and reduces myocardial infarction size following I hour of myocardial ischemia and 24 hours of reperfusion.

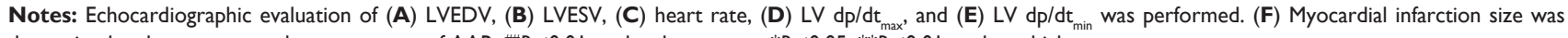
determined and was expressed as percentage of AAR. ${ }^{\# P} P 0.01$ vs the sham group; $* P<0.05, * * P<0.01$ vs the vehicle group.

Abbreviations: LVEDV, left ventricular end diastolic volume; LVESV, left ventricular end systolic volume; LV $\mathrm{dp}_{\mathrm{dt}} \mathrm{dmax}_{\text {, }}$ left ventricular pressure maximal rate of rise; $\mathrm{LV} \mathrm{dp/dt} \mathrm{min}_{\text {in }}$, left ventricular pressure maximal rate of decline; $A A R$, area at risk. 

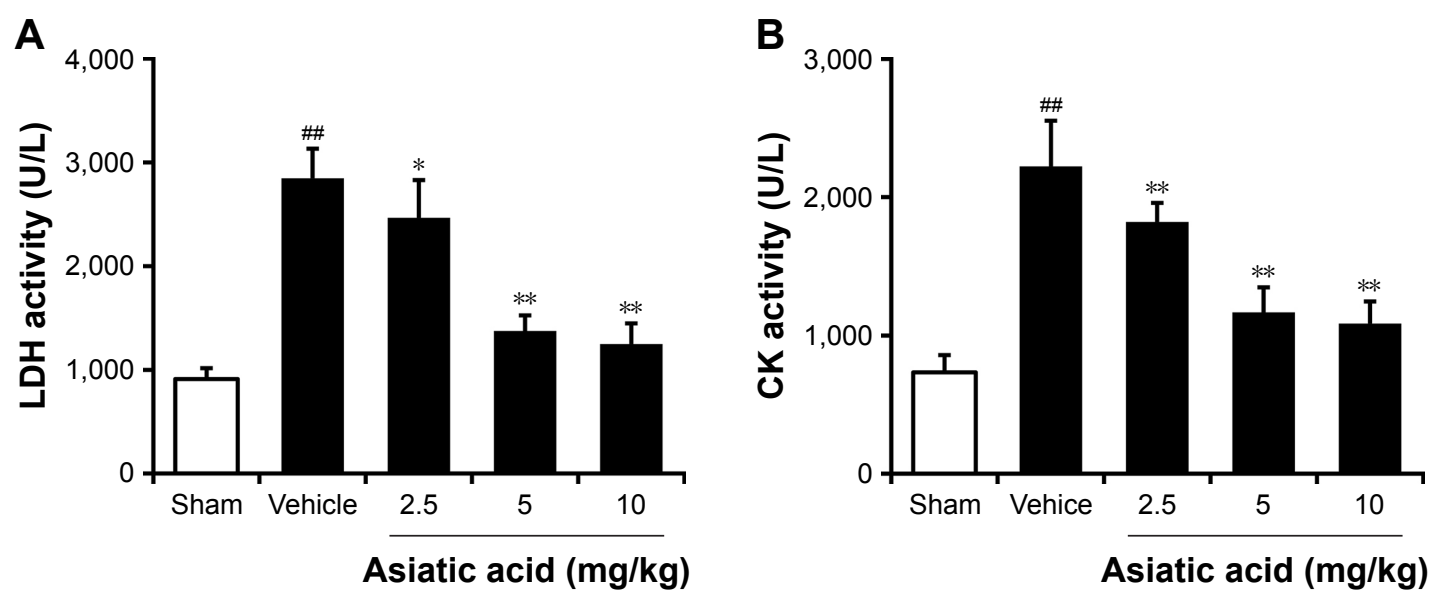

Figure 2 Asiatic acid reduced plasma LDH and CK activities following MI/R.

Notes: (A) LDH and (B) CK activities were measured after I hour of myocardial ischemia and 24 hours of reperfusion. ${ }^{\#} P<0.0$ I vs the sham group; $* P<0.05$, $* * P<0.0$ I vs the vehicle group.

Abbreviations: LDH, lactate dehydrogenase; CK, creatine kinase; MI/R, myocardial ischemia/reperfusion.

CK activity, which was also suppressed by different doses of asiatic acid (Figure 2B).

\section{Asiatic acid prevents cardiomyocyte apoptosis after $\mathrm{MI} / \mathrm{R}$}

Cardiomyocyte apoptosis was detected in rats subjected to 1 hour of myocardial ischemia followed by 24 hours of reperfusion. In sham rats, few TUNEL-positive cells were observed in randomly selected fields. MI/R resulted in a marked increase in cardiomyocyte apoptosis. By contrast, different doses of asiatic acid significantly reduced the percentage of apoptotic cells after MI/R injury (Figure 3A). Quantitative analysis of TUNEL-positive cells in each group is shown in Figure 3B. These results suggested that asiatic acid effectively prevents cardiomyocyte apoptosis after MI/R.

\section{Asiatic acid activates Akt/GSK-3 $\beta$ signal pathway following $\mathrm{MI} / \mathrm{R}$}

Our previous work has shown that asiatic acid attenuates oxygen-glucose deprivation/reoxygenation-induced myocardial apoptosis through activation of Akt/GSK-3 $\beta$ pathway. ${ }^{11}$ Herein, we further investigated whether asiatic
A
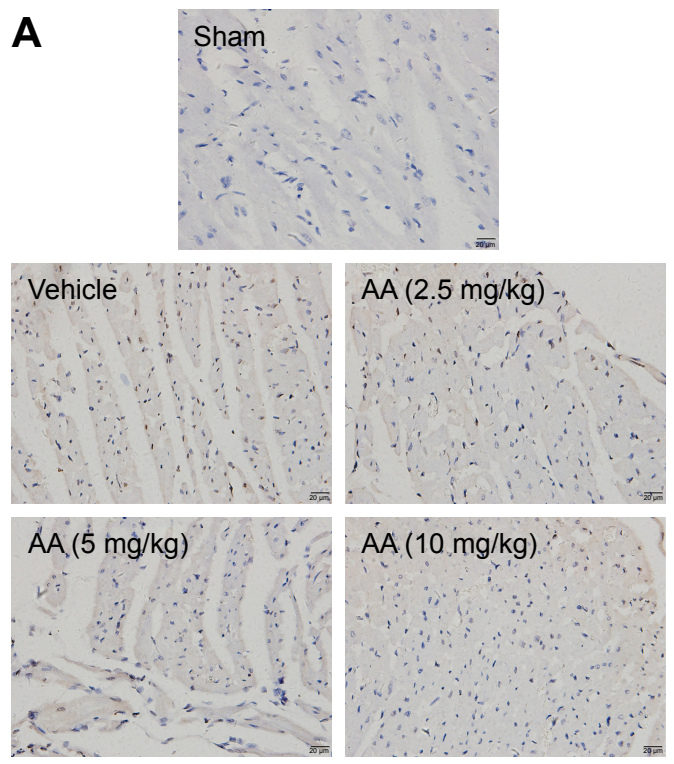

$\mathrm{AA}(10 \mathrm{mg} / \mathrm{kg})$

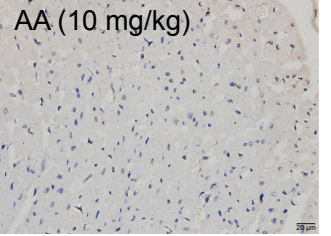

B

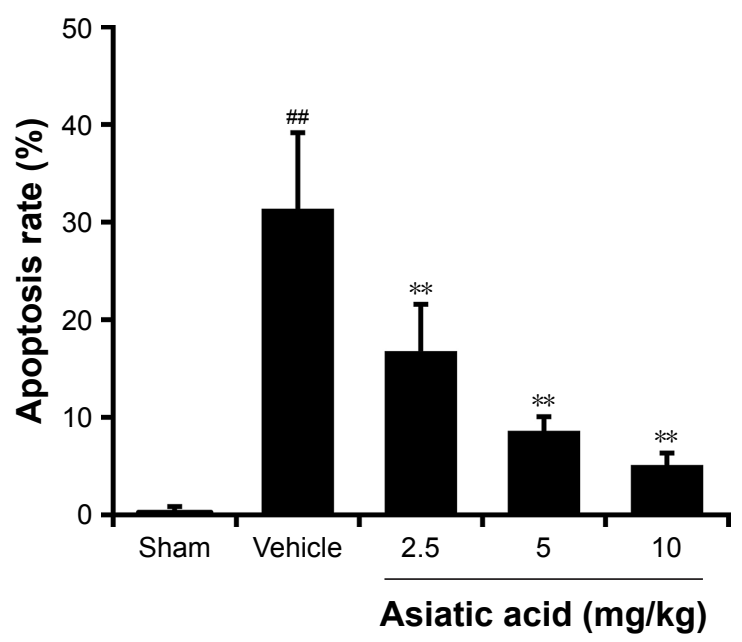

Figure 3 Asiatic acid prevents cardiomyocyte apoptosis after I hour of myocardial ischemia and 24 hours of reperfusion.

Notes: (A) Representative images of TUNEL assay in rat myocardium. Scale bar, $20 \mu \mathrm{m}$. (B) Quantitative analysis of TUNEL-positive cells in each group. ${ }^{\# P}<0.0$ I vs the sham group; ${ }^{*} P<0.01$ vs the vehicle group.

Abbreviation: AA, asiatic acid. 
acid has the same effects on Akt/GSK-3 $\beta$ activation in the animal model. MI/R caused an increase in phosphor-Akt levels, which was further upregulated by asiatic acid treatment at 5 and $10 \mathrm{mg} / \mathrm{kg}$ (Figure 4A). GSK-3 $\beta$ phosphorylation at Ser9 was also induced after MI/R and asiatic acid significantly promoted GSK-3 $\beta$ phosphorylation compared with the vehicle group (Figure 4B). In accordance with the results of in vitro experiments, asiatic acid promotes the activation of the Akt/GSK-3 $\beta$ signaling pathway following MI/R in vivo.

\section{Asiatic acid suppresses glycogen breakdown and maintains glucose homeostasis in ischemic rats through}

\section{Akt activation}

The effects of asiatic acid on glycometabolism in ischemic myocardium was assessed by measuring the glycogen content as well as the plasma glucose and lactate concentrations. The dose of asiatic acid showing the maximum cardioprotection was used and LY294002, a PI3K/Akt inhibitor, was employed to explore the underlying mechanism of asiatic acid-mediated cardioprotection. The glycogen content in the ischemic myocardium was measured at 1-hour period of ischemia and at 24 hours of reperfusion, respectively. Myocardial glycogen stores significantly dropped in the ischemic myocardium, with $\sim 51.4 \%$ reduction at 1 hour time point and
$85.6 \%$ reduction at 24 hours time point, respectively. While asiatic acid treatment significantly prevented glycogen breakdown, especially at the end of 1-hour ischemia, these effects were abolished in the presence of LY294002 (Figure 5A).

The plasma glucose increased at 1 hour of ischemia and recovered to normal levels at 24 hours of reperfusion. Asiatic acid lowered plasma glucose levels during the ischemic period, while LY294002 reversed its hypoglycemic effect (Figure 5B). The plasma lactate concentration elevated during ischemia and peaked at 24 hours reperfusion. Asiatic acid significantly blunted the release of lactate to plasma, which was increased by the combination with LY294002 treatment (Figure 5C). Taken together, asiatic acid modulates glycometabolism during $\mathrm{MI} / \mathrm{R}$ in an Akt-dependent manner.

\section{Asiatic acid increases PPAR $\gamma$ levels and promotes GLUT4 translocation in ischemic rats}

Since asiatic acid exhibited a beneficial effect on glycometabolism during ischemia/reperfusion, we hypothesized several targets, including PPAR $\gamma$ and GLUT4, that are involved in asiatic acid's action of regulating glycometabolism. Realtime PCR analysis showed that asiatic acid treatment induced a 4.58-fold increase in PPAR $\gamma$ mRNA levels, whereas the addition of LY294002 did not alter PPAR $\gamma \mathrm{mRNA}$ expression
A
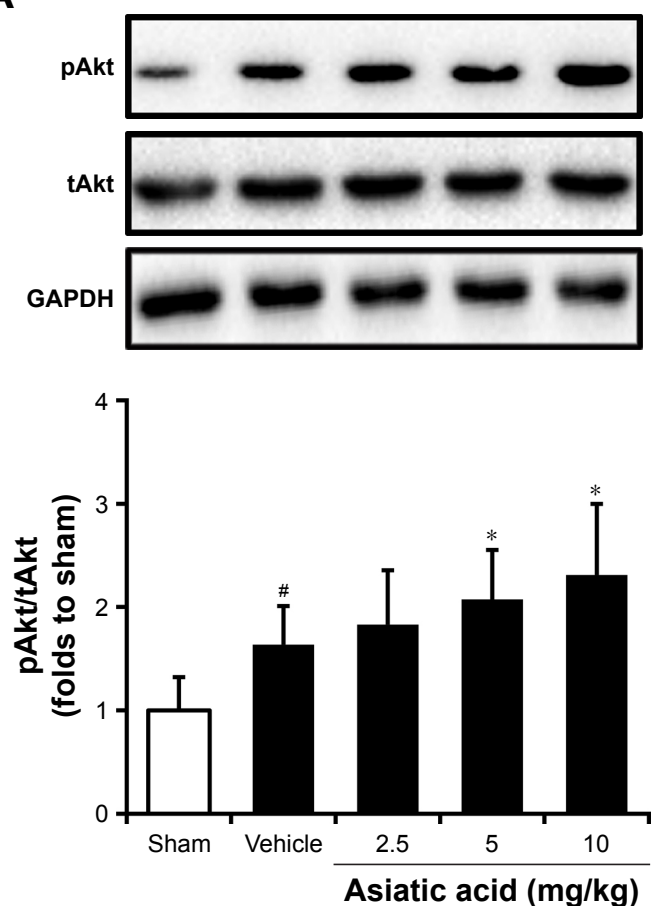

B
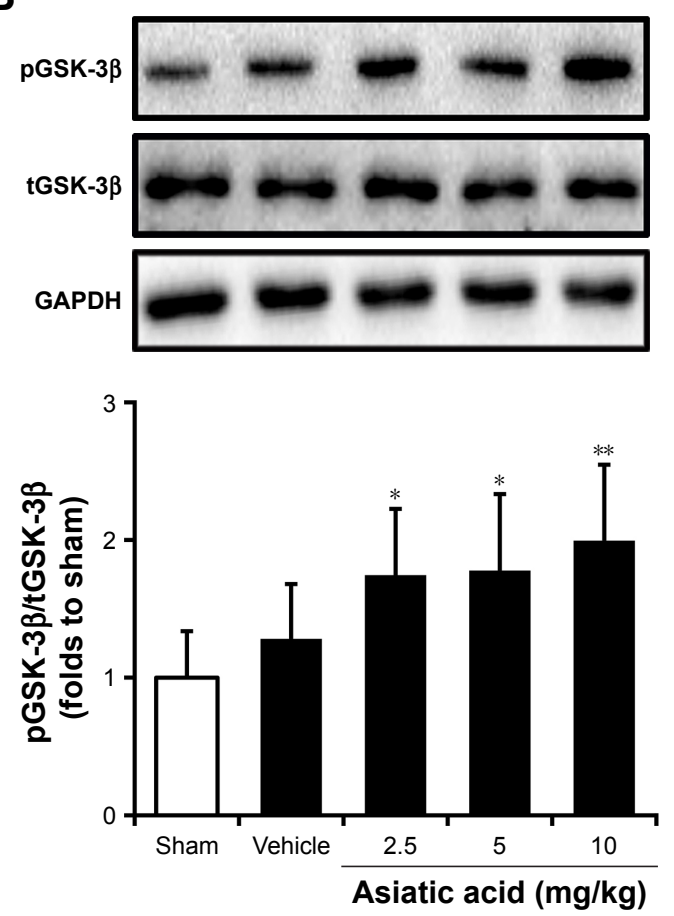

Figure 4 Asiatic acid activates Akt/GSK-3 $\beta$ signal pathway following I hour of myocardial ischemia and 24 hours of reperfusion.

Notes: (A) Phosphor-Akt (pAkt) and total Akt (tAkt) levels determined by Western blot and quantitative analysis of pAkt levels normalized to tAkt levels. (B) Phosphor-

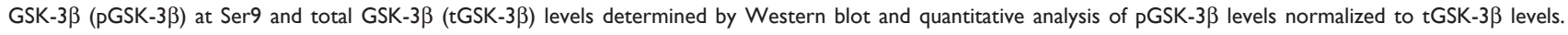
${ }^{\sharp} P<0.05$ vs the sham group; $* P<0.05, * * P<0.0$ I vs the vehicle group. 
A

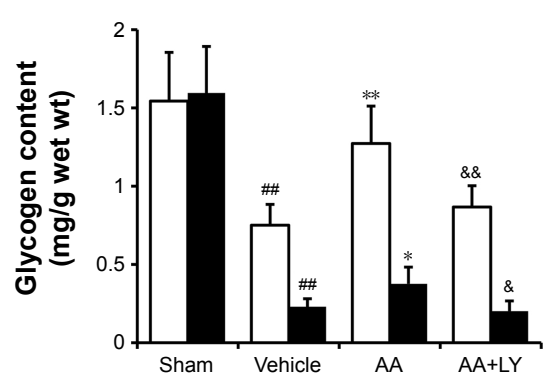

B

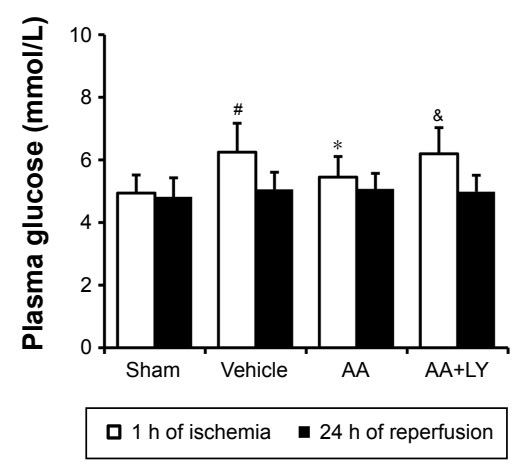

C

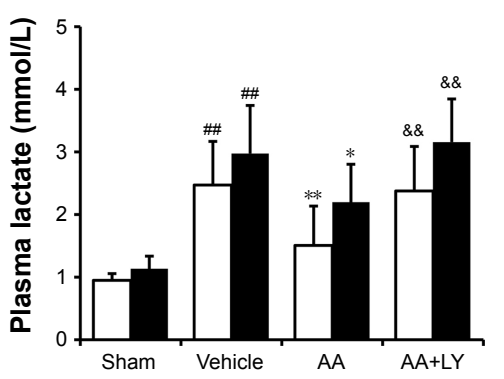

Figure 5 Asiatic acid improves glycometabolism in ischemic rats through Akt activation.

Notes: (A) Glycogen content, (B) plasma glucose, and (C) plasma lactate were determined in sham, vehicle, asiatic acid (AA), and asiatic acid combined with LY294002 $(A A+L Y)$ groups at the end of I hour of myocardial ischemia and after 24 hours of reperfusion, respectively. ${ }^{\#}<<0.05$, ${ }^{\#} P<0.0$ I vs the sham group; ${ }^{*} P<0.05, * * P<0.0$ I vs the vehicle group; ${ }^{\&} P<0.05,{ }^{\text {\&\&}} P<0.0$ I vs the asiatic acid-treated group.

compared with the single treatment of asiatic acid under normal conditions. MI/R resulted in a decrease in PPAR $\gamma$ expression which was reversed by asiatic acid treatment. In addition, LY294002 did not affect PPAR $\gamma$ expression when combined with asiatic acid treatment (Figure 6A). The mRNA levels of GLUT4 did not change significantly in each group before and after MI/R (Figure 6B). Asiatic acid treatment or asiatic acid combined with LY294002 significantly enhanced the protein levels of PPAR $\gamma$ in sham group and prevented PPAR $\gamma$ downregulation after ischemia/reperfusion injury compared with the vehicle group (Figure 6C and D). Asiatic acid did not change total GLUT4 levels but potently promoted its translocation to membrane both under normal condition and following ischemia/reperfusion injury. However, when LY294002 was concurrently administered with asiatic acid, it attenuated the augmented GLUT4 translocation (Figure 6C and E). These data confirmed that asiatic acid promotes PPAR $\gamma$ expression in an Akt-independent manner
A

C

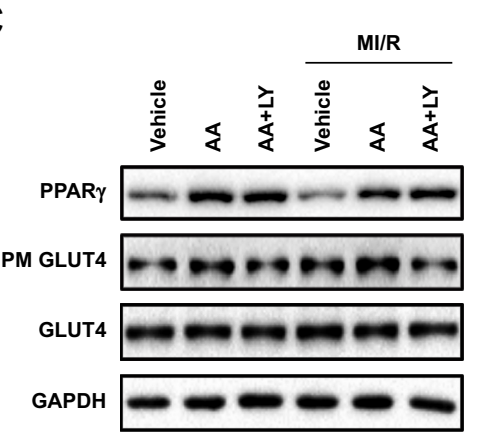

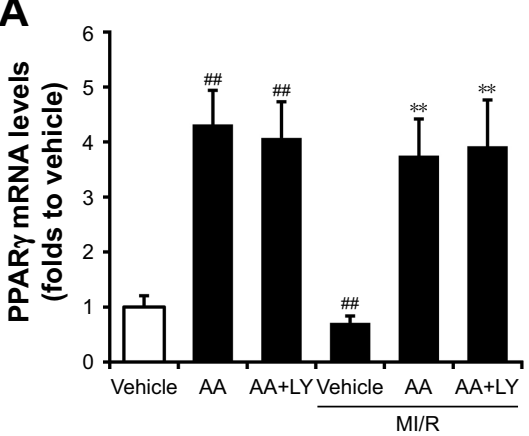

D

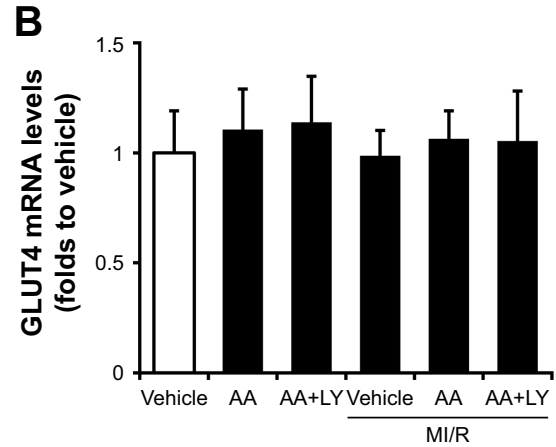

E

Figure 6 Asiatic acid increases PPAR $\gamma$ levels and promotes GLUT4 translocation to plasma membrane in ischemic rats.

Notes: The myocardium was isolated from vehicle-, asiatic acid (AA)-, and asiatic acid plus LY294002 (AA+LY)-treated rats under normal condition and following I hour of myocardial ischemia and 24 hours of reperfusion, respectively. The mRNA levels of (A) PPAR $\gamma$ and (B) GLUT4 were determined by real-time PCR. (C) PPAR $\gamma$, GLUT4 from plasma membrane (PM GLUT4), GLUT4, and GAPDH levels were determined by Western blot. (D) Quantitative analysis of PPAR $\gamma$ levels were normalized to GAPDH levels, and (E) GLUT4 translocation levels (PM GLUT4) were normalized to total GLUT4. ${ }^{*} P<0.05,{ }^{\# P} P<0.0$ I vs the sham group; ${ }^{* * P}<0.0$ I vs the vehicle group; ${ }^{\text {\&\&}} P<0.0$ I vs the asiatic acid-treated group.

Abbreviation: MI/R, myocardial ischemia/reperfusion. 
while it ameliorates the translocation of GLUT4 from the cytoplasm to the PM through Akt activation.

\section{Discussion}

Here, we demonstrated that pretreatment with asiatic acid significantly ameliorated the MI/R injury, including improvement of the cardiac function and serologic indicators, reduction of myocardial infarction size, and inhibition of cardiomyocyte apoptosis. Mechanistically, asiatic acid suppressed MI/R-induced glycogen breakdown, inhibited the elevation of plasma glucose and lactate concentrations, and promoted the translocation of GLUT4 to PM through Akt/GSK-3 $\beta$ activation. In addition, asiatic acid treatment increased PPAR $\gamma$ expression at both mRNA and protein levels after ischemia/reperfusion insult.

The cardiac glycogen is a potential source of myocardial energy under aerobic and anaerobic conditions. During myocardial ischemia when exogenous glucose delivery is reduced, myocardial glycogen and glycogenolysis are important sources of glycolytic substrate. ${ }^{16}$ Previously studies have shown that elevated glycogen levels in heart have cardioprotective effects against ischemic injury. ${ }^{17,18}$ However, the rapid consumption of glycogen resulting from the anaerobic metabolism causes excessive production of lactic acid and metabolic acidosis. ${ }^{19}$ Therefore, it is of great significance to inhibit glycogenolysis and maintain glycogen content in ischemic myocardium, and pharmacological intervention of these processes would be cardioprotective from ischemia/ reperfusion injury.

Glycogen content in cells and tissue depends on the concerted regulation of glycogen synthesis by glycogen synthase (GS) and glycogen breakdown by GP through an intricate network of signal transduction pathways. ${ }^{20} \mathrm{GP}$ is a homodimeric enzyme existing in a phosphorylated $(\mathrm{GPa})$ and an unphosphorylated form $(\mathrm{GPb})$, and it has three isoforms named after the tissues where it is dominantly expressed: liver (pygl), brain (pygb), and muscle (pygm). ${ }^{21}$ The inhibition of GP might suppress glucose production arising from glycogenolysis and gluconeogenesis, ${ }^{20}$ and recent studies showed that GP inhibitors have cardioprotective effects. ${ }^{8}$ A serious pentacyclic triterpenes have been identified as GP inhibitors and developed as hypoglycemic agents. ${ }^{7,22,23}$ We have previously demonstrated that maslinic acid, a pentacyclic triterpenoid compound, inhibits GP activity in homogenates of cultured astrocytes ${ }^{24}$ and reduces cerebral ischemic injury in hyperglycemic rats. ${ }^{25}$ Asiatic acid is another pentacyclic triterpene showing hypoglycemic activity in different diabetic animal models. ${ }^{10,26,27}$ We have observed that asiatic acid effectively prevents cardiomyocyte apoptosis after oxygen-glucose deprivation/ reperfusion injury. ${ }^{11}$ Taken together, we hypothesized that asiatic acid may protect against ischemia/reperfusion injury by modulation of glycometabolism in cardiomyocyte. In the present study, we found that asiatic acid inhibited glycogen decomposition, stabilized plasma glucose levels, and reduced lactic acid release during $\mathrm{MI} / \mathrm{R}$, with reduced myocardial infarction size and myocardial apoptosis. These results indicated that interfering with glycometabolism in cardiacmyocyte is an effective way to attenuate MI/R injury.

Glycogen synthase kinase-3 (GSK-3), a serine/threonine protein kinase, plays a crucial role in numerous cellular processes including glycogen metabolism. GSK-3 is one of the rate-limiting enzymes in glycogen synthesis and glucose transport, and also acts as an important signaling molecule in the Akt-GSK-3 $\beta$ pathway. ${ }^{28}$ The activation of Akt-GSK-3 $\beta$ leads to the phosphorylation of GSK-3 $\beta$ at ser9, which promotes the transformation of glycogen synthase to the active form, thereby increasing glycogen synthesis. ${ }^{29}$ The glucose transporters GLUT4 are mainly responsible for glucose intake from the blood vessels. The increase of GLUT4 activity and the translocation of GLUT4 to the cell membrane during ischemia can improve the efficiency of glucose utilization. ${ }^{30}$ Therefore, we examined the role of Akt-GSK-3 $\beta$ signal pathway as well as GLUT4 in ischemic myocardium. As expected, asiatic acid enhanced Akt phosphorylation and resulted in inactivation of GSK-3 $\beta$. The total levels of GLUT4 mRNA and protein remained unchanged, but the amount of GLUT4 on PM was markedly increased after asiatic acid treatment following MI/R, suggesting an enhancement of glucose transport function. The use of the Akt inhibitor further confirmed the participation of Akt in asiatic acid-mediated improvement of glycometabolism and glucose transport.

PPAR $\gamma$ is a ligand-activated transcription factor, which has been certified as an important regulator of glucose homeostasis and inflammation. ${ }^{31}$ PPAR $\gamma$ regulates a variety of biological processes within the cardiovascular system, and activation of PPAR $\gamma$ exhibits cardioprotective effects in myocardial ischemia and diabetic cardiomyopathy. ${ }^{32-34}$ Previous data showed that compounds from the leaves of Salacia oblonga exhibited enhanced uptake and utilization of glucose through dual activation of PPAR $\gamma$ and GLUT4. ${ }^{35}$ Recently, a series of pentacyclic triterpenes and their derivatives have been identified as potential PPAR $\gamma$ agonists. ${ }^{36}$ Asiatic acid was previously shown to directly upregulate PPAR $\gamma$ levels in human gingival fibroblasts. ${ }^{37}$ In an in vivo model of hepatic ischemia/reperfusion, asiatic acid activates PPAR $\gamma / \mathrm{NLRP} 3$ signaling pathway to mitigate hepatic histopathological 
damage and global inflammatory level. ${ }^{12}$ According to these findings, it is speculated that asiatic acid may act on PPAR $\gamma$ in ischemic myocardium. We demonstrated here a significant increase in PPAR $\gamma$ mRNA and protein levels after asiatic acid treatment. Myocardial ischemia induced a decrease in PPAR $\gamma$ expression, which was reversed in asiatic acid-treated rats. Asiatic acid combined with the Akt inhibitor LY294002 did not affect PPAR $\gamma$ levels, indicating that asiatic acid-mediated PPAR $\gamma$ activation is Akt-independent, and possibly functions as an upstream signal of Akt as previously reported..$^{38}$ This might be a new mechanism of asiatic acid for the protective effects in cardiomyopathy. In addition, recent evidence has revealed that the phosphorylation on PPAR $\gamma$ is vital for the regulation of its activity and can influence its physiological functions including adipogenesis, lipid metabolism, and insulin sensitivity. ${ }^{39-41}$ However, the exact roles of phospho-PPAR $\gamma$ in asiatic acid-mediated glucose homeostasis and cardioprotection remain unclear and warrant further investigation in our lab, and the use of PPAR $\gamma$ antagonist would further confirm the effect of asiatic acid on PPAR $\gamma$ activation.

Increased PPAR $\gamma$ expression accompanied with activated Akt signaling and increased membrane translocation of GLUT4 was observed in a number of studies, and compounds that promote PPAR $\gamma$ expression also contribute to the activation of Akt/GLUT4 pathway. ${ }^{42-44}$ Considering that PPAR $\gamma$, Akt, and GLUT4 are all associated with insulin signaling and sensitivity, an upstream regulator may exist to modulate their function and interaction. Further research to elucidate the underlying mechanisms by which asiatic acid modulates PPAR $\gamma$, Akt, and GLUT4 is also necessary.

In summary, asiatic acid exerts protection against MI/R injury through inhibition of glycogen breakdown and stabilization of plasma glucose and lactate levels. These effects may be mediated by Akt/GSK-3 $\beta$ activation and thereby promoting GLUT4 translocation to plasma membrane, and also by PPAR $\gamma$ upregulation. The findings may provide novel evidence for the prevention and treatment of MI/R injury by using GP inhibitors.

\section{Acknowledgment}

This work was supported by the National Natural Science Foundation of China (grant 81400220).

\section{Author contributions}

All authors contributed toward data analysis, drafting, and revising the paper and agree to be accountable for all aspects of the work.

\section{Disclosure}

The authors report no conflicts of interest in this work.

\section{References}

1. Roger VL, Go AS, American Heart Association Statistics Committee and Stroke Statistics Subcommittee, et al. Heart disease and stroke statistics - 2012 update: a report from the American Heart Association. Circulation. 2012;125(1):e2-e220.

2. Jaswal JS, Keung W, Wang W, Ussher JR, Lopaschuk GD. Targeting fatty acid and carbohydrate oxidation - a novel therapeutic intervention in the ischemic and failing heart. Biochim Biophys Acta. 2011;1813(7): 1333-1350.

3. King LM, Opie LH. Glucose and glycogen utilisation in myocardial ischemia - changes in metabolism and consequences for the myocyte. Mol Cell Biochem. 1998;180(1-2):3-26.

4. Murphy E, Steenbergen C. Mechanisms underlying acute protection from cardiac ischemia-reperfusion injury. Physiol Rev. 2008; 88(2):581-609.

5. Goyard D, Kónya B, Chajistamatiou AS, et al. Glucose-derived spiroisoxazolines are anti-hyperglycemic agents against type 2 diabetes through glycogen phosphorylase inhibition. Eur J Med Chem. 2016; 108:444-454.

6. Baker DJ, Timmons JA, Greenhaff PL. Glycogen phosphorylase inhibition in type 2 diabetes therapy: a systematic evaluation of metabolic and functional effects in rat skeletal muscle. Diabetes. 2005;54(8):2453-2459.

7. Leonidas DD, Hayes JM, Kato A, et al. Phytogenic Polyphenols as Glycogen Phosphorylase Inhibitors: The Potential of Triterpenes and Flavonoids for Glycaemic Control in Type 2 Diabetes. Curr Med Chem. 2017;24(4):384-403.

8. Tracey WR, Treadway JL, Magee WP, et al. Cardioprotective effects of ingliforib, a novel glycogen phosphorylase inhibitor. Am J Physiol Heart Circ Physiol. 2004;286(3):H1177-H1184.

9. Wen X, Sun H, Liu J, et al. Naturally occurring pentacyclic triterpenes as inhibitors of glycogen phosphorylase: synthesis, structureactivity relationships, and X-ray crystallographic studies. J Med Chem. 2008;51(12):3540-3554.

10. Wang X, Lu Q, Yu DS, et al. Asiatic acid mitigates hyperglycemia and reduces islet fibrosis in Goto-Kakizaki rat, a spontaneous type 2 diabetic animal model. Chin J Nat Med. 2015;13(7):529-534.

11. Huang X, Zuo L, Lv Y, et al. Asiatic Acid Attenuates Myocardial Ischemia/Reperfusion Injury via Akt/GSK-3 $\beta /$ HIF- $1 \alpha$ Signaling in Rat H9c2 Cardiomyocytes. Molecules. 2016;21(9):E1248:1248.

12. Xu Y, Yao J, Zou C, et al. Asiatic acid protects against hepatic ischemia/reperfusion injury by inactivation of Kupffer cells via PPAR $\gamma /$ NLRP3 inflammasome signaling pathway. Oncotarget. 2017; 8(49):86339-86355.

13. Yu L, Li Z, Dong X, et al. Polydatin Protects Diabetic Heart against Ischemia-Reperfusion Injury via Notch1/Hes1-Mediated Activation of Pten/Akt Signaling. Oxid Med Cell Longev. 2018;2018:2750695.

14. Zacharowski K, Olbrich A, Otto M, Hafner G, Thiemermann C. Effects of the prostanoid EP3-receptor agonists M\&B 28767 and GR 63799X on infarct size caused by regional myocardial ischaemia in the anaesthetized rat. Br J Pharmacol. 1999;126(4):849-858.

15. Zhang QJ, Li QX, Zhang HF, et al. Swim training sensitizes myocardial response to insulin: role of Akt-dependent eNOS activation. Cardiovasc Res. 2007;75(2):369-380.

16. Fraser H, Lopaschuk GD, Clanachan AS. Assessment of glycogen turnover in aerobic, ischemic, and reperfused working rat hearts. Am J Physiol. 1998;275(5 Pt 2):H1533-H1541.

17. Omar MA, Wang L, Clanachan AS. Cardioprotection by GSK-3 inhibition: role of enhanced glycogen synthesis and attenuation of calcium overload. Cardiovasc Res. 2010;86(3):478-486.

18. Ke Z, Wang G, Yang L, et al. Crude terpene glycoside component from Radix paeoniae rubra protects against isoproterenol-induced myocardial ischemic injury via activation of the PI3K/AKT/mTOR signaling pathway. J Ethnopharmacol. 2017;206:160-169. 
19. Saddik M, Lopaschuk GD. Myocardial triglyceride turnover and contribution to energy substrate utilization in isolated working rat hearts. J Biol Chem. 1991;266(13):8162-8170.

20. Gerich JE. Control of glycaemia. Baillieres Clin Endocrinol Metab. 1993;7(3):551-586.

21. Newgard CB, Hwang PK, Fletterick RJ. The family of glycogen phosphorylases: structure and function. Crit Rev Biochem Mol Biol. 1989;24(1):69-99.

22. Liu J, Zhang H, Zhu P, et al. Synthesis and biological evaluation of ambradiolic acid as an inhibitor of glycogen phosphorylase. Fitoterapia. 2015;100:50-55.

23. Liu J, Wang X, Chen YP, et al. Maslinic acid modulates glycogen metabolism by enhancing the insulin signaling pathway and inhibiting glycogen phosphorylase. Chin J Nat Med. 2014;12(4):259-265.

24. Guan T, Li Y, Sun H, Tang X, Qian Y. Effects of maslinic acid, a natural triterpene, on glycogen metabolism in cultured cortical astrocytes. Planta Med. 2009;75(10):1141-1143.

25. Guan T, Qian Y, Tang X, et al. Maslinic acid, a natural inhibitor of glycogen phosphorylase, reduces cerebral ischemic injury in hyperglycemic rats by GLT-1 up-regulation. J Neurosci Res. 2011;89(11): 1829-1839.

26. Sun W, Xu G, Guo X, et al. Protective effects of asiatic acid in a spontaneous type 2 diabetic mouse model. Mol Med Rep. 2017;16(2): 1333-1339.

27. Ramachandran V, Saravanan R. Glucose uptake through translocation and activation of GLUT4 in PI3K/Akt signaling pathway by asiatic acid in diabetic rats. Hum Exp Toxicol. 2015;34(9):884-893.

28. Macaulay K, Woodgett JR. Targeting glycogen synthase kinase-3 (GSK-3) in the treatment of Type 2 diabetes. Expert Opin Ther Targets. 2008;12(10):1265-1274.

29. Patel S, Doble BW, Macaulay K, Sinclair EM, Drucker DJ, Woodgett JR. Tissue-specific role of glycogen synthase kinase 3 beta in glucose homeostasis and insulin action. Mol Cell Biol. 2008;28(20):6314-6328.

30. Taegtmeyer H. Ischemia and glucose metabolism. Circulation. 1997;96(10):3810-3811.

31. Murphy GJ, Holder JC. PPAR-gamma agonists: therapeutic role in diabetes, inflammation and cancer. Trends Pharmacol Sci. 2000; 21(12):469-474.

32. Chen K, Li D, Zhang X, Hermonat PL, Mehta JL. Anoxia-reoxygenation stimulates collagen type-I and MMP-1 expression in cardiac fibroblasts: modulation by the PPAR-gamma ligand pioglitazone. J Cardiovasc Pharmacol. 2004;44(6):682-687.
33. Nissen SE, Wolski K. Effect of rosiglitazone on the risk of myocardial infarction and death from cardiovascular causes. $N$ Engl J Med. 2007;356(24):2457-2471

34. Mahajan UB, Chandrayan G, Patil CR, et al. The Protective Effect of Apigenin on Myocardial Injury in Diabetic Rats mediating Activation of the PPAR- $\gamma$ Pathway. Int J Mol Sci. 2017;18(4):E756.

35. Singh AK, Raj V, Keshari AK, et al. Isolated mangiferin and naringenin exert antidiabetic effect via PPAR/GLUT4 dual agonistic action with strong metabolic regulation. Chem Biol Interact. 2018;280:33-44.

36. Zhang L, Dong J, Liu J, et al. Synthesis and biological evaluation of novel pentacyclic triterpene derivatives as potential PPAR $\gamma$ agonists. Med Chem. 2013;9(1):118-125

37. Hao C, Wu B, Hou Z, et al. Asiatic acid inhibits LPS-induced inflammatory response in human gingival fibroblasts. Int Immunopharmacol. 2017;50:313-318.

38. Kilter H, Werner M, Roggia C, et al. The PPAR-gamma agonist rosiglitazone facilitates Akt rephosphorylation and inhibits apoptosis in cardiomyocytes during hypoxia/reoxygenation. Diabetes Obes Metab. 2009;11(11):1060-1067.

39. Choi JH, Banks AS, Estall JL, et al. Anti-diabetic drugs inhibit obesity-linked phosphorylation of PPAR $\gamma$ by Cdk5. Nature. 2010; 466(7305):451-456.

40. Choi JH, Banks AS, Kamenecka TM, et al. Antidiabetic actions of a non-agonist PPAR $\gamma$ ligand blocking Cdk5-mediated phosphorylation. Nature. 2011;477(7365):477-481.

41. Rangwala SM, Rhoades B, Shapiro JS, et al. Genetic modulation of PPARgamma phosphorylation regulates insulin sensitivity. Dev Cell. 2003;5(4):657-663.

42. Gandhi GR, Stalin A, Balakrishna K. Insulin sensitization via partial agonism of PPAR $\gamma$ and glucose uptake through translocation and activation of GLUT4 in PI3K/p-Akt signaling pathway by embelin in type 2 diabetic rats. Biochim Biophys Acta. 2013;1830(1):2243-2255.

43. Tandrasasmita OM, Wulan DD, Nailufar F, Sinambela J, Tjandrawinata RR. Glucose-lowering effect of DLBS3233 is mediated through phosphorylation of tyrosine and upregulation of PPAR $\gamma$ and GLUT4 expression. Int J Gen Med. 2011;4:345-357.

44. Gandhi GR, Jothi G, Antony PJ, et al. Gallic acid attenuates high-fat diet fed-streptozotocin-induced insulin resistance via partial agonism of PPAR $\gamma$ in experimental type 2 diabetic rats and enhances glucose uptake through translocation and activation of GLUT4 in PI3K/p-Akt signaling pathway. Eur J Pharmacol. 2014;745:201-216.
Drug Design, Development and Therapy

\section{Publish your work in this journal}

Drug Design, Development and Therapy is an international, peerreviewed open-access journal that spans the spectrum of drug design and development through to clinical applications. Clinical outcomes, patient safety, and programs for the development and effective, safe, and sustained use of medicines are the features of the journal, which

\section{Dovepress}

has also been accepted for indexing on PubMed Central. The manuscript management system is completely online and includes a very quick and fair peer-review system, which is all easy to use. Visit http://www.dovepress.com/testimonials.php to read real quotes from published authors. 